\title{
Application of the $\mathrm{k}-\omega$ turbulence model to assess the flutter derivatives of a long span bridge
}

\author{
F. Brusiani ${ }^{1}$, G. Cazzoli ${ }^{1}$, S. de Miranda ${ }^{2}$, F. Ubertini $^{2}$ \& P. Vaona ${ }^{2}$ \\ ${ }^{1}$ DIEM Department, Bologna University, Italy \\ ${ }^{2}$ DICAM Department, Bologna University, Italy
}

\begin{abstract}
This paper considers the influence of the turbulence model adopted to numerically predict the aero-elastic phenomena in Fluid Structure Interaction (FSI) problems. After a detailed evaluation of the main suitable turbulence models available in the Reynolds Average Navier Stokes (RANS) approach, the two-equation k- $\omega$ turbulent model was identified as the best compromise between solution accuracy and computational cost. In order to test the k- $\omega$ turbulence model performance for FSI applications, a specific simulation methodology was defined. It was firstly tested in assessing the aerodynamic forces resulting on static bluff bodies for which experimental databases were available. Then, the simulation methodology based on the k- $\omega$ turbulence model was used to reproduce the steady and unsteady aerodynamic forces induced on a Great Belt East bridge cross section subjected to wind load. In particular, the unsteady aerodynamic forces induced on the bridge were evaluated by calculating the flutter derivatives. As for the test cases, also for the Great Belt East bridge the computational results were compared to experimental evidence obtained by wind tunnel tests.
\end{abstract}

Keywords: numerical simulation, turbulence, long span bridge, flutter derivatives.

\section{Introduction}

On built environments subjected to fluid flow action, vibrations can be forced by vortex resonance or structure self-excitation. When fluid-structure interaction 
(FSI) happens, all built environment members (structural and non-structural) must be carefully designed in order to avoid dramatic events as happened to the first Tacoma Narrows Bridge which collapsed under wind action in the first part of the previous century. To estimate the tendency of a body to vibrate under fluid flow load, two ways can be followed: wind tunnel test and numerical simulation.

Due to its flexibility, during the last years the computational approach has gained importance with respect to the traditional experimental investigation. Tamura [1], Tamura and Itoh [2], Tamura and Ono [3], Vairo [4], and more recently Huang et al. [5] well presented how the FSI computations could be proficiently applied in wind engineering field. However, still today, the numerical simulation of FSI problems is not as wider adopted as could be expected. The main reason of that resides in the difficulties to numerically predict the fully turbulent, unsteady, and 3D flow conditions generated around more-or-less bluff bodies immersed in a fluid flow. Because of such a flow conditions, to include turbulence representation in FSI numerical analysis is a must. It returns the choice of turbulence model as the key point to capture the right turbulent time and length scales associated to the flow.

In the literature, many papers deal with different strategies about how to manage the turbulence in FSI problems, spanning from Large Eddy Simulation (LES) to laminar simulation. About LES $[1-3,6]$, this is a very sophisticated and physically grounded method whose use is limited by its high computational cost. On the other hand, the computational cost of the laminar approach [7] is low but its application range in FSI field is quite limited because it returns a physical representation of the flow only at very low Reynolds numbers. Between these two methods, Reynolds Average Navier Stokes (RANS) approach surely represents the today's most used approach to perform FSI computations. By the RANS approach, the physics of a turbulent flow can be predicted at an affordable computational cost because the turbulent flow around the generic body is not directly solved but its effects are superimposed on the mean flow adopting a specific turbulence model.

The aim of this paper was to show the primary role played by the turbulence closure model to improve the prediction on motion induced aerodynamic forces on bluff bodies. In this context, the authors decided to deeply test the $\mathrm{k}-\omega$ turbulence model capabilities in FSI application field. This paper demonstrates how the two-equation $\mathrm{k}-\omega$ turbulence model could be successfully used to predict the motion induced aerodynamic forces on bluff bodies. First evidences about this topic can be found in [8].

Starting from the k- $\omega$ turbulence model choice, a simulation methodology was set to evaluate the static and dynamic forces imposed on a cross section of the Great Belt East suspended bridge immersed in a fluid flow. In particular, the dynamic forces were evaluated by calculating the flutter derivatives. The resultant predictions were compared with experimental results as well as other numerical results presented in similar works by other authors.

All the simulations referred in this paper were performed by using the Computational Fluid Dynamic (CFD) commercial code Fluent v6.3 [9]. 


\section{Numerical method}

\subsection{Turbulence model}

In RANS, the time-averaging operation on the governing equations throw away all details concerning the state of the flow contained in the instantaneous fluctuations, therefore all the turbulent scales need to be modelled by a closure model. Among all the closure models available in RANS, the most popular are the two-equation $k-\varepsilon[10]$ and $k-\omega$ models $[10,11]$. These two models are very cheap in terms of computing resources. The main difference between them lies in the near wall flow solution. The k- $\varepsilon$ model does not allow the direct integration through the boundary layer because $\varepsilon$ falls to zero near the wall. Moreover, k- $\varepsilon$ over-estimates the turbulent kinetic energy in stagnation regions near the walls. On the other hand, the k- $\omega$ model allows the direct integration through the boundary layer permitting to improve the wall layer solution as demonstrated in [11]. This is a very important feature for FSI computational applications because the near wall flow solution is quite important to locate the laminar/turbulent transition point in the boundary layer, therefore to determine the overall flow topological structure around the body immersed into the flow. For this reason, in authors' opinion the $\mathrm{k}-\omega$ model is well suited for FSI computations. In this paper, the k- $\omega$ model proposed by Wilcox [11] was considered. The turbulence kinetic energy $(\mathrm{k})$ and the specific dissipation rate $(\omega)$ are obtained by solving the following transport equations:

$$
\begin{gathered}
\frac{\partial}{\partial t}(\rho k)+\frac{\partial}{\partial x_{i}}\left(\rho k u_{i}\right)=\frac{\partial}{\partial x_{j}}\left(\Gamma_{k} \frac{\partial k}{\partial x_{j}}\right)+G_{k}-Y_{k}+S_{k} \\
\frac{\partial}{\partial t}(\rho \omega)+\frac{\partial}{\partial x_{i}}\left(\rho \omega u_{i}\right)=\frac{\partial}{\partial x_{j}}\left(\Gamma_{\omega} \frac{\partial \omega}{\partial x_{j}}\right)+G_{\omega}-Y_{\omega}+S_{\omega}
\end{gathered}
$$

where $G_{k}$ represents the generation of turbulence kinetic energy due to mean velocity gradients, $G_{\omega}$ represents the generation of $\omega, \Gamma_{\mathrm{k}}$ and $\Gamma_{\omega}$ represent the effective diffusivity of $k$ and $\omega$ respectively, $Y_{k}$ and $Y_{\omega}$ represent the dissipation of $k$ and $\omega$ due to turbulence, $S_{k}$ and $S_{\omega}$ are source terms. It should be underlined as the RANS k- $\omega$ turbulence model assumes isotropic turbulence, therefore the $3 \mathrm{D}$ vortical structures in the span wise direction are lose. This means that $3 \mathrm{D}$ simulation gives the same solution as from $2 \mathrm{D}$ simulation. In view of this, $2 \mathrm{D}$ simulation approach can be used to reduce the computational cost without compromising accuracy.

\subsection{Flutter derivatives}

Flutter derivatives are empirical parameters that can be used to calculate the motion-induced forces on bluff-bodies immersed in a fluid flow. In general, the aerodynamic forces acting on a bluff body can be expressed in this form: 


$$
L=\frac{1}{2} \rho U^{2} B C_{L}(t) \quad D=\frac{1}{2} \rho U^{2} B C_{D}(t) \quad M=\frac{1}{2} \rho U^{2} B^{2} C_{M}(t)
$$

where the induced lift force (L), drag force (D), and pitching momentum (M) are expressed as a function of mean incoming flow velocity $U$, flow density $\rho$, body width $B . \mathrm{C}_{\mathrm{L}}(\mathrm{t}), \mathrm{C}_{\mathrm{D}}(\mathrm{t})$, and $\mathrm{C}_{\mathrm{M}}(\mathrm{t})$ are the instantaneous lift, drag, and pitching momentum non-dimensional coefficients.

According to Scanlan and Simiu [13], the lift induced aerodynamic force and the pitching momentum can be expressed as linear combination of body motions:

$$
\begin{aligned}
& L=\frac{1}{2} \rho U^{2} B\left(K H_{1}^{*} \frac{\dot{h}}{U}+K H_{2}^{*} \frac{B \dot{\alpha}}{U}+K^{2} H_{3}^{*} \alpha+K^{2} H_{4}^{*} \frac{h}{B}\right) \\
& M=\frac{1}{2} \rho U^{2} B^{2}\left(K A_{1}^{*} \frac{\dot{h}}{U}+K A_{2}^{*} \frac{B \dot{\alpha}}{U}+K^{2} A_{3}^{*} \alpha+K^{2} A_{4}^{*} \frac{h}{B}\right)
\end{aligned}
$$

where $h, \dot{h}, \alpha$ and $\dot{\alpha}$ are the vertical and torsional displacements and their time derivatives, $K=\omega B / U$ is the reduced oscillation frequency, and $\omega=2 \pi f$ is the oscillation circular frequency. For the drag force a formulation similar to the lift can be written [13]. It is not reported here for brevity.

Coefficients $H_{i}{ }^{*}$ and $A_{i}{ }^{*}$ in eqns. (4) and (5) are the so called dimensionless flutter derivatives that must be calculated (experimentally or computationally) to evaluate the tendency of a body to vibrate under the flow action. To extract the flutter derivatives of a rigid body, a vertical $\left(h=h_{0} \cdot e^{i \omega t}\right)$ or torsional $\left(\alpha=\alpha_{0} \cdot e^{i \omega t}\right)$ harmonic motion has to be forced on it. By the least square method, the stable $\mathrm{C}_{\mathrm{L}}(\mathrm{t})$ and $\mathrm{C}_{\mathrm{M}}(\mathrm{t})$ profiles are fitted obtaining their amplitudes $\left(\mathrm{C}_{\mathrm{L}}, \mathrm{C}_{\mathrm{M}}\right)$ and the phases $\left(\delta_{\mathrm{L}}, \delta_{\mathrm{M}}\right)$. Considering eqns. (3), (4), and (5) for the vertical or torsional motion law, the aero-elastic derivatives can be written in these forms:

$$
\frac{B K^{2} C_{L}\left(\cos \delta_{L}-i \sin \delta_{L}\right)}{8 \pi^{2} h_{0}}=i H_{1}^{*}+H_{4}^{*} ; \frac{B K^{2} C_{M}\left(\cos \delta_{M}-i \sin \delta_{M}\right)}{8 \pi^{2} h_{0}}=i A_{1}^{*}+A_{4}^{*}
$$

for the vertical motion and

$$
\frac{K^{2} C_{L}\left(\cos \delta_{L}-i \sin \delta_{L}\right)}{8 \pi^{2} \alpha_{0}}=i H_{2}^{*}+H_{3}^{*} ; \frac{K^{2} C_{M}\left(\cos \delta_{M}-i \sin \delta_{M}\right)}{8 \pi^{2} \alpha_{0}}=i A_{2}^{*}+A_{3}^{*}
$$

for the torsional motion.

\section{Computed flow field results}

To validate the two-equation $\mathrm{k}-\omega$ model in predicting the motion induced aerodynamic forces on a bluff-body, the computational campaign was subdivided into two steps. Firstly, the k- $\omega$ model capabilities were tested to predict the steady aerodynamic forces resulting on two classic bodies immersed 
in a fluid flow. Secondly, the k- $\omega$ model capabilities were exploited to predict both steady and unsteady aerodynamic forces induced on a bridge cross section by wind action.

On the basis of the remarks given in the previous sections, all the RANS simulations were performed in 2D. Air at ambient temperature and pressure was considered as incompressible working fluid.

\subsection{Test cases}

As test cases two simple cross section shapes were considered: circular cross section [4, 12] and rectangular cross section [8]. During this analysis phase, no motions were imposed on the bodies. In both cases, a segregated flow solver was adopted together with a first-order implicit Euler scheme [14]. Central Differencing (CD) scheme [10,14] and UpWind second-order scheme [10,14] were considered to discretize diffusive and convective fluxes respectively. The pressure-velocity coupling was achieved by means of the SIMPLE algorithm.

\subsubsection{Circular cross section}

A circular cross-section of diameter $\mathrm{D}$ was placed into a fluid domain having a length of $36 \mathrm{D}$ and a height of 17D. The domain was discretized by a nonstructured mesh of about 132000 hexahedral cells. The cell height close to the section surface was equal to $1.6 \cdot 10^{-3} \mathrm{D}$. On the inlet boundary, the Reynolds number was set to $1 \cdot 10^{4}$ (defined on the basis of D) and the inflow turbulence intensity was assumed equal to $5 \%$.

Fig. 1 shows the time-traces of dimensionless drag and lift coefficients recorded during the simulation. Their mean values were compared with the experimental ones as reported in table 1. Also the Stroual number St corresponding to the periodic flow behaviour around the cross section $(\mathrm{St}=\mathrm{n} \cdot \mathrm{D} / \mathrm{U}$
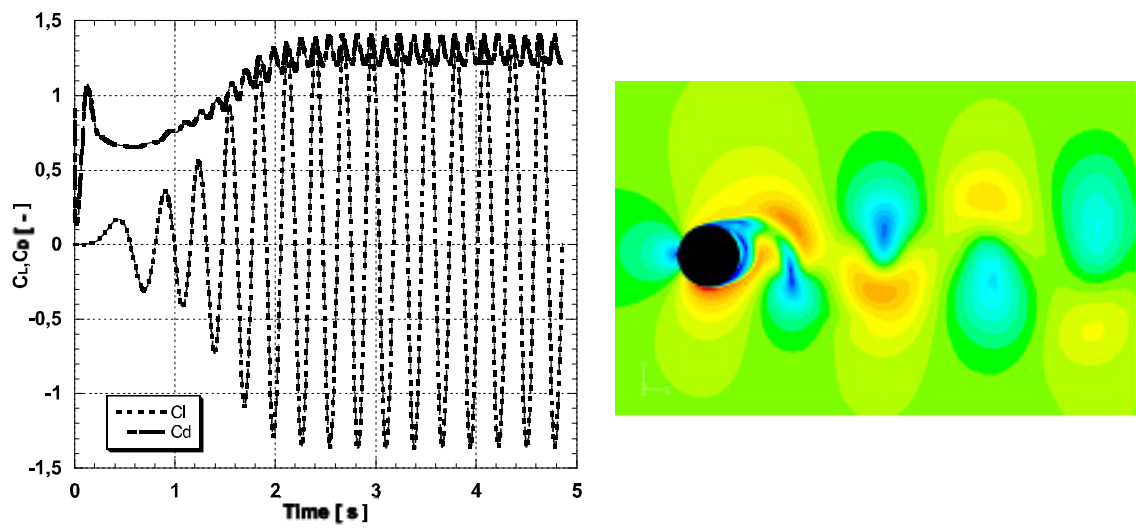

Figure 1: Numerical lift and drag coefficients time-traces for the circular cross-section. 
where $\mathrm{n}$ is the vortex-shedding frequency), was calculated and compared with experimental evidence. As showed, the agreement between experimental and computational results was quite good demonstrating the turbulence model capability to correctly capture the shear layer dynamics.

Table 1: Mean force coefficients and Stroual number obtained testing the circular cross-section.

\begin{tabular}{|c|c|c|c|}
\hline & $\mathrm{C}_{\mathrm{L}}$ & $\mathrm{C}_{\mathrm{D}}$ & $\mathrm{St}$ \\
\hline Exp. & $0 \pm 1.05$ & $1.2 \pm 0.27$ & 0.27 \\
\hline Num. & $0 \pm 1.33$ & $1.3 \pm 0.1$ & 0.26 \\
\hline
\end{tabular}

\subsubsection{Rectangular cross section}

A rectangular cross section having an aspect ratio L/B equal to 4 was considered ( $\mathrm{L}$ was the section dimension along the main flow direction). The bluff body was placed into a computational domain having length and height equal to $12 \mathrm{~L}$ and $4 \mathrm{~L}$ respectively. The computational domain was discretized by about 117000 hexahedral cells. The cell height near the surface was equal to $1.3 \cdot 10^{-3} \mathrm{~L}$. On the inlet boundary, the Reynolds number was set to $2.54 \cdot 10^{4}$ (defined on the basis of B) and the inflow turbulence intensity was assumed equal to $1 \%$.

Fig. 2 depicts the time-traces of dimensionless drag and lift coefficients recorded during the simulation. Table 2 compares their mean values and the Stroual value with the experimental ones. As experienced about the circular cross section, also in this case the agreement between experimental and computational results was quite good.
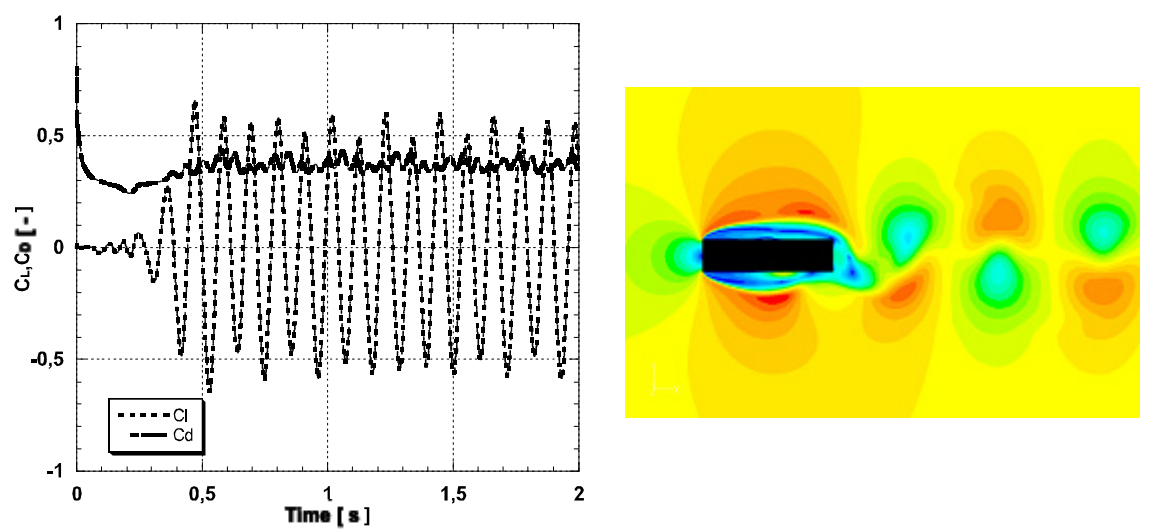

Figure 2: Numerical lift and drag coefficients time-traces for the rectangular cross-section. 
Table 2: Mean force coefficients and Stroual number obtained testing the rectangular cross-section.

\begin{tabular}{|c|c|c|c|}
\hline & $\mathrm{C}_{\mathrm{L}}$ & $\mathrm{C}_{\mathrm{D}}$ & $\mathrm{St}$ \\
\hline Exp. & $-0.07 \pm 0.29$ & $0.356 \pm 0.08$ & 0.140 \\
\hline Num. & $0 \pm 0.35$ & $0.350 \pm 0.042$ & 0.145 \\
\hline
\end{tabular}

\subsection{Great Belt East bridge}

The simulation results obtained by the previous test cases highlighted the effectiveness of the simulation methodology concerning the possibility to numerically predict the flow separation and the near-wall law around generic bodies.

To complete the analysis of the $\mathrm{k}-\omega$ model effectiveness for FSI applications, the cross section of the Great Belt East Bridge built in Denmark was considered (fig. 3). No accessory structures (e.g. guard rails) were modelled. The computational results were compared to the experimental database obtained by wind tunnel tests performed on a bridge section model (scale 1:80) at Danish Maritime Institute [15]. To perform the computations, the scaled bridge cross section was immersed into a computational domain having length and height equal to $12 \mathrm{~B}$ and $5.5 \mathrm{~B}$ respectively (B was the deck length).
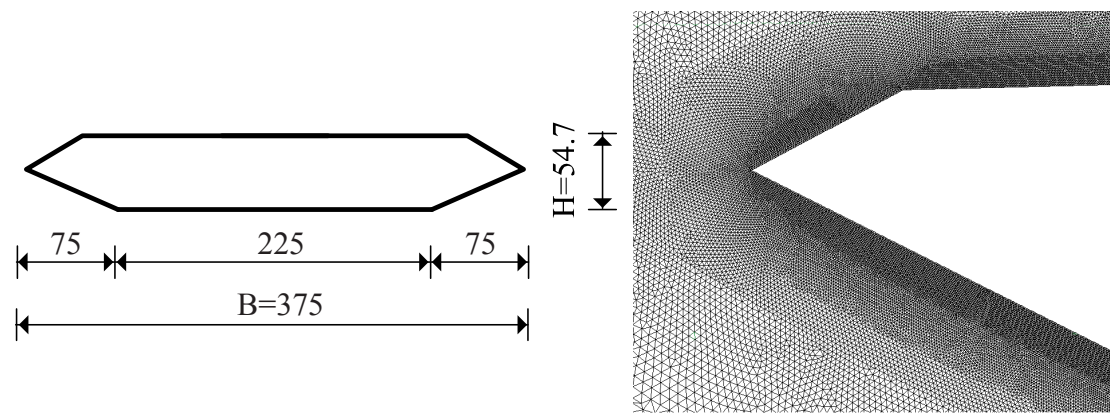

Figure 3: $\quad$ Shape of the Great Belt East cross-section (scale 1:80, dimensions in $\mathrm{mm}$ ) and adopted mesh structure.

The computational campaign performed on the bridge deck was subdivided into two stages named stationary and dynamic. During the stationary stage, the steady aerodynamic forces acting on the fixed bridge cross section were evaluated by the dimensionless lift and momentum mean coefficients calculated for five values of the incidence angle. During the dynamic stage, vertical and rotational harmonic motions were separately imposed on the bridge cross section centre of gravity. The resulting unsteady aerodynamic forces acting on the bridge section were evaluated through the flutter derivatives.

For both stationary and dynamic analyses, the same non-structured mesh was adopted (fig. 3). The grid size was about 313000 hexahedral/tetrahedral cells. 
The mesh size in the bridge wall proximity was defined to maintain the $\mathrm{y}^{+}$[7] value close to 3 along all the section profile.

\subsubsection{Conditions of computation}

To perform the Great Belt East simulation, a velocity inlet condition and an outflow condition were applied on the left and right sides of the computational box respectively. On domain top and bottom sides symmetry conditions were imposed. The Reynolds number of the coming flow was set equal to $1.1 \mathrm{e}+5$. According to standard wind tunnel turbulent conditions, a turbulent intensity of $0.5 \%$ and a turbulent viscosity ratio of 2.0 were applied to both the inlet and outlet boundaries.

The same numerical schemes adopted for the previous test cases were adopted also for the bridge analysis. The simulation time step was chosen to maintain the Courant number (Co) under the value of 1.5 over all of the computational domain. According to Dugué et al.'s investigation [16], this Co limit was chosen as the best compromise between solution quality and computational cost.

\subsubsection{Great East Belt: stationary aerodynamic analysis}

The steady aerodynamic forces acting on the bridge were evaluated for five values of the incidence angle: $0^{\circ}, \pm 5^{\circ}$, and $\pm 10^{\circ}$ (for all cases, the cross-section had a fixed position into the flow box.).

For each case, the dimensionless mean coefficients $C_{L}$ and $C_{D}$ were calculated starting from the time-traces recorded during the simulations. For example, fig. 4 shows the $\mathrm{C}_{\mathrm{L}}$ and $\mathrm{C}_{\mathrm{D}}$ time-traces recorded during the computation performed for the incidence angle of $+5^{\circ}$. The comparison between experiments and computations (fig. 5) revealed that the proposed simulation methodology was able to guarantee a good prediction of the static bridge aerodynamic performance for all the considered configurations. The predicted Stroual number for $0^{\circ}$ incidence angle was equal to 0.14 , in good agreement with the experimental range [7].
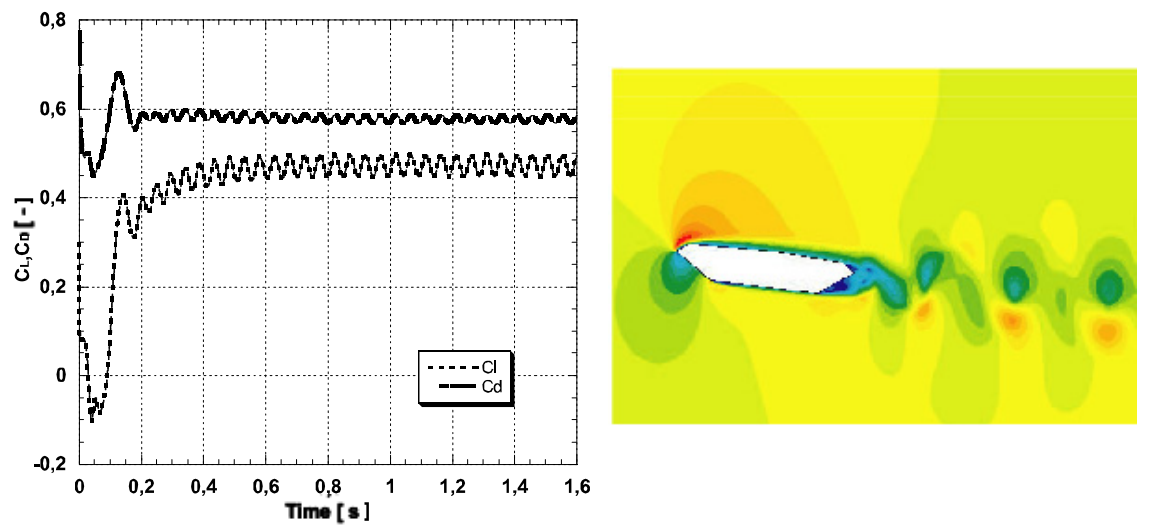

Figure 4: Numerical lift and drag coefficient time-traces for the Great East Belt cross-section at incidence angle equal to $+5^{\circ}$. 


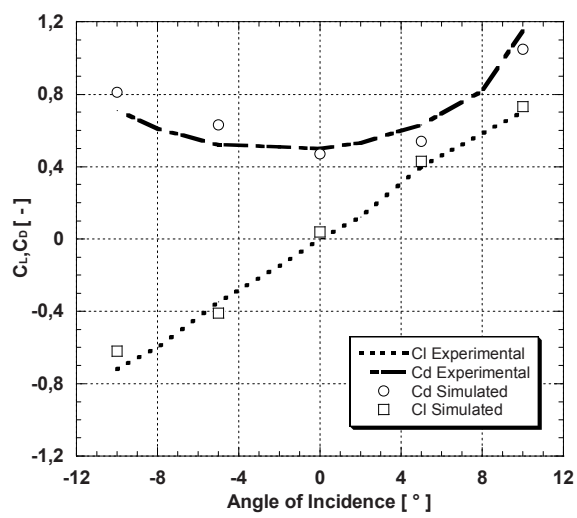

Figure 5: Comparison between experimental and computational steady aerodynamic coefficients for the Great East Belt against the angle of incidence.

\subsubsection{Great East Belt: dynamic aerodynamic analysis}

Based on the simulation methodology previously presented, the flutter derivatives were calculated applying separately two harmonic motions on the Great East Belt rigid cross-section: a vertical motion having an amplitude of $0.025 \mathrm{~m}$ and a torsional motion having an amplitude of $3^{\circ}$. For each harmonic motion, four reduced velocities were considered: $3,5,7$, and 11 (the reduced velocity is defined as $U /(f \cdot B)$ ).

On the basis of authors' experience and evidences presented in other papers [5], to manage the mesh motion of the bridge section the computational domain was split into three parts: rigid motion zone, deforming zone, and static zone (fig. 6). The rigid motion zone was directly connected to the rigid bridge profile, therefore the vertical/torsional motion applied to the cross-section was identically applied to all the mesh nodes into the rigid motion zone. The static zone was not deformed by the bridge-section motion. The deforming zone

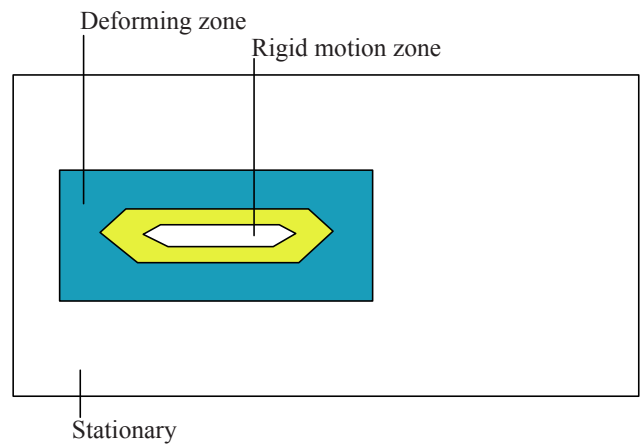

Figure 6: Computational domain subdivision to manage the mesh motion. 
represented the only mesh region deformed by the bridge motion at each time step. Adopting this strategy, the best initial mesh quality assured into the boundary layer region (rigid motion zone) at the simulation starting point was not reduced by the cross-section motion.

Regarding the mesh structure, the whole computational domain was covered by triangular elements. In this way, the mesh stretch into the deforming zone could be handled using two algorithms specifically designed to work only on tetrahedral cells: spring-based smoothing algorithm and remeshing algorithm [9]. During the dynamic analysis of the Great East Belt, at each time step the mesh internal nodes placed between the rigid motion zone and the static zone were repositioned according to the bridge displacement. After the node displacements, each cell was checked by quality parameters based on the cell dimension and deformation. The cells that did not satisfy the quality criteria were marked and then remeshed before updating the solution to the next time step.

3.2.3.1 Computed flow field For the harmonic vertical motion case, fig. 7 shows the time history for lift and momentum obtained for a reduced velocity of 11. From the mathematical elaboration of these instantaneous profiles, the $\left(\mathrm{H}_{1}{ }^{*}\right.$, $\left.\mathrm{H}_{4}{ }^{*}\right)$ and $\left(\mathrm{A}_{1}{ }^{*}, \mathrm{~A}_{4}{ }^{*}\right)$ values were extracted (eqn. (6)). As showed in fig. 8, the flutter derivatives numerical predictions were fairly close to the experimental ones. Moreover, comparing the present results with the flutter derivatives obtained in [5] by using the k- $\varepsilon$ model, it was possible to observe the quite evident improvement guaranteed by changing the turbulence model. Probably the observed improvements could be mainly associated to the higher solution accuracy reached into the boundary layer region thanks to $k-\omega$ model features.
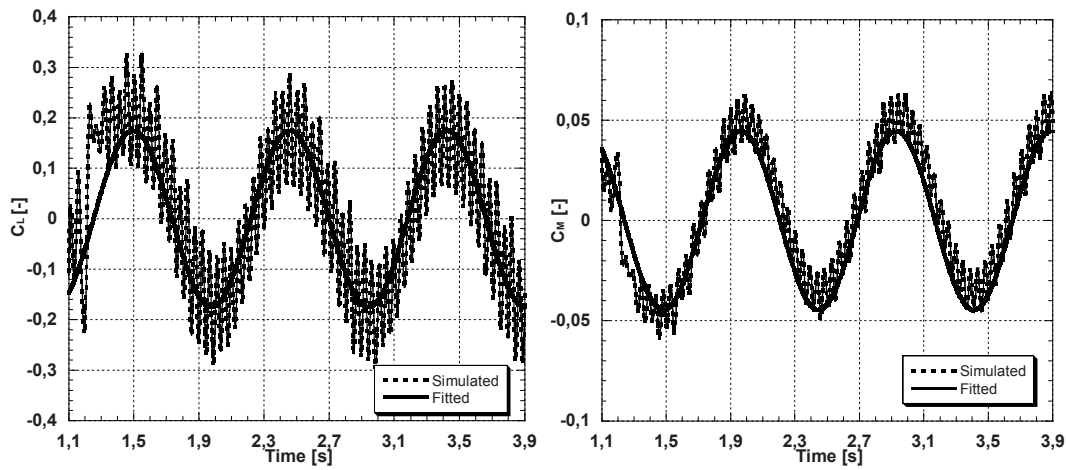

Figure 7: Great east belt non-dimensional lift and momentum coefficients recorded for a wind reduced velocity equal to 11 (vertical motion).

The same behaviour was observed also for the torsional motion. Fig. 8 shows the $\left(\mathrm{H}_{2}{ }^{*}, \mathrm{H}_{3}{ }^{*}\right)$ and $\left(\mathrm{A}_{2}{ }^{*}, \mathrm{~A}_{3}{ }^{*}\right)$ values obtained for the considered reduced velocity values. Also in this case, the computational results obtained by the $\mathrm{k}-\omega$ model were quite close to both experimental and $\mathrm{k}-\varepsilon$ results. 
Maybe the large part of the differences remaining between experimental and computational results could be ascribed to the accessory structure effects that were not considered in the present analysis.
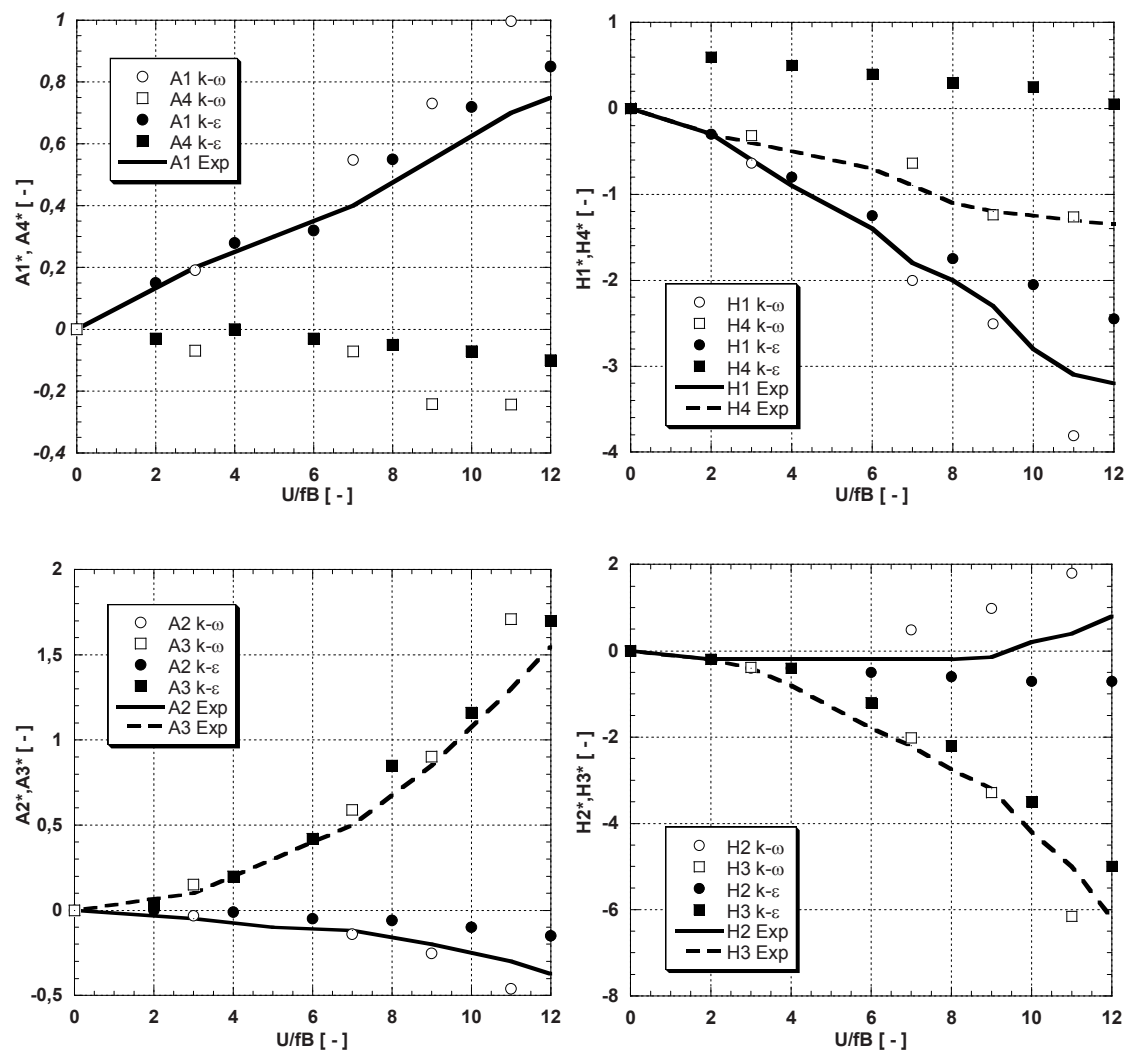

Figure 8: Flutter derivatives of the great east belt cross section.

\section{Conclusions}

The aim of this work was to verify the potentiality of the k- $\omega$ turbulence model for FSI applications. To do that a simulation methodology was defined and firstly tested on two benchmarks to evaluate the k- $\omega$ capabilities in predicting the static aerodynamic forces on them. Then, the same simulation methodology was adopted to calculate static and dynamic behaviour of a Great East Belt rigid cross section under the wind action. In particular, the dynamic behaviour of the bridge section was evaluated by calculating the flutter derivatives. In all the considered cases, physically grounded solutions in good agreement with experiments were obtained. In particular, the $\mathrm{k}-\omega$ turbulence model led to improve the flutter derivatives evaluations with respect to simulations methodology based on the k- $\varepsilon$ turbulence model. 


\section{References}

[1] Tamura, T., Reliability on CFD estimation for wind-structure interaction problems. J. Wind. Eng. Ind. Aerodyn. 81, pp. 117-143, 1999.

[2] Tamura, T., Itoh, Y., Unstable oscillation of rectangular cylinder at various mass ration. J. Aerospace Eng. 12(4), pp. 136-144, 1999.

[3] Tamura, T., Ono, Y., LES analysis on aeroelastic instability of prisms in turbulent flow. J. Wind. Eng. Ind. Aerodyn. 91, pp. 1827-1846, 2003.

[4] Vairo, G., A numerical model for wind loads simulation on long-span bridges. Simulation Modelling Practice and Theory 11, pp. 315-351, 2003.

[5] Huang, L., Haili, L., Bin, W., Yongle, L., Numerical simulation for aerodynamic derivatives of bridge deck. Simulation Modelling Practice and Theory 17, pp. 719-729, 2009.

[6] Sun, D., Owen, J.S., Wright, N.G., Liaw, K., Fluid-structure interaction of prismatic line-like structures using LES and block-iterative coupling. $J$. Wind. Eng. Ind. Aerodyn. 96, pp. 840-858, 2008.

[7] Frandsen, J.B., Numerical bridge deck studies using finite elements. Part I: flutter. J. Fluids Struct. 19, pp. 171-191, 2004.

[8] Sun, D., Owen, J.S., Wright, N.G., Application of the $k$ - $\omega$ turbulence model for a wind-induced vibration study of 2D bluff bodies. J. Wind. Eng. Ind. Aerodyn. 97, pp. 77-87, 2009.

[9] Fluent 6.3, Fluent Inc. 2006, Lebanon, NH, USA.

[10] Versteeg, H. K., Malalasekera, W., (eds). An Introduction to Computational Fluid Dynamics: The Finite Volume Method (2nd edition), Longman, 2006.

[11] Wilcox, D.C., Turbulence Modeling for CFD, DCW Industries Inc., La Canada, CA, USA, 1998.

[12] Schlighting, H., Boundary Layer Theory, McGraw-Hill, 1979.

[13] Scanlan, R.H., Simiu, E., (eds). Wind Effects on Structures, third ed., John Wiley and Sons Inc., 1996.

[14] Ferziger, J. H., Peric, M., (eds). Computational Methods for Fluid Dynamics, Springer, 2001.

[15] Reinhold, T.A., Brinch, M., Damsgaard, A., Wind tunnel tests for the Great Belt Link. Proc. Of the $1^{\text {st }}$ Int. Symposium on Aerodynamics of Large Bridges, eds. Larsen, A., Copenhagen, Denmark, 1992.

[16] Dugué, V., Gauchet, N., and Veynante, D., Applicability of Large Eddy Simulation to the Fluid Mechanics in a Real Engine Configuration by Means of an Industrial Code. LES for Piston Engine Flows Workshop, IFP, Paris 2006. 